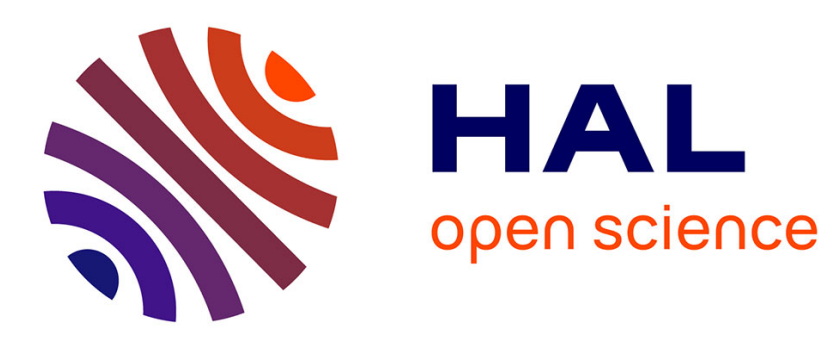

\title{
Assessing human brain impedance using simultaneous surface and intracerebral recordings
}

Radu Ranta, Steven Le Cam, Louise Tyvaert, Valérie Louis-Dorr

\section{To cite this version:}

Radu Ranta, Steven Le Cam, Louise Tyvaert, Valérie Louis-Dorr. Assessing human brain impedance using simultaneous surface and intracerebral recordings. Neuroscience, 2017, 343, pp.411-422. 10.1016/j.neuroscience.2016.12.013 . hal-01429285

\section{HAL Id: hal-01429285 \\ https://hal.science/hal-01429285}

Submitted on 7 Jan 2017

HAL is a multi-disciplinary open access archive for the deposit and dissemination of scientific research documents, whether they are published or not. The documents may come from teaching and research institutions in France or abroad, or from public or private research centers.
L'archive ouverte pluridisciplinaire HAL, est destinée au dépôt et à la diffusion de documents scientifiques de niveau recherche, publiés ou non, émanant des établissements d'enseignement et de recherche français ou étrangers, des laboratoires publics ou privés. 


\title{
Assessing human brain impedance using simultaneous surface and intracerebral recordings
}

\author{
Radu Ranta $^{\mathrm{a}, \mathrm{b}, *}$, Steven Le Cam ${ }^{\mathrm{a}, \mathrm{b}}$, Louise Tyvaert ${ }^{\mathrm{a}, \mathrm{b}, \mathrm{c}}$, Valérie Louis-Dorr ${ }^{\mathrm{a}, \mathrm{b}}$ \\ ${ }^{a}$ Université de Lorraine, CRAN, UMR 7039, 54500 Vandoeuvre-lès-Nancy, France \\ ${ }^{b}$ CNRS, CRAN, UMR 7039, France \\ ${ }^{c}$ CHU Nancy, Neurology Department, 54000 Nancy, France
}

\begin{abstract}
Most of the literature on the brain impedance proposes a frequency independent resistive model. Recently, this conclusion was tackled by a series of papers (Bédard et al., 2006; Bédard and Destexhe, 2009; Gomes et al., 2016), based on microscopic scale modelling and measurements. Our paper aims to investigate the impedance issue using simultaneous in vivo depth and surface signals recorded during intracerebral electrical stimulation of epileptic patients, involving a priori different tissues with different impedances. Our results confirm the conclusions from (Logothethis et al., 2007): there is no evidence of frequency dependence of the brain tissue impedance (more precisely, there is no difference, in terms of frequency filtering, between the brain and the skull bone), at least at a macroscopic scale. In order to conciliate findings from both microscopic and macroscopic scales, we recall different neural/synaptic current generators models from the literature and we propose an original computational model, based on fractional dynamics.
\end{abstract}

Keywords: Brain impedance; simultaneous EEG-SEEG; intracerebral stimulation; computational current source models

\footnotetext{
*Corresponding author

Email address: radu.ranta@univ-lorraine.fr (Radu Ranta)
} 


\section{INTRODUCTION}

In brain electro-physiology, the widely accepted model considers currents sources embedded in the brain tissue and potential measurements using electrodes, either implanted in the brain (micro-electrodes, SEEG), placed on the brain surface $(\mathrm{ECoG})$ or on the scalp (EEG). Different scales can be considered for the sources and, consequently, for the measurements, from membrane patches and single cells to synchronized neuronal populations. At the microscopic scale, current sources are considered to be the ionic channels generating sub-threshold activities or action potentials. At the macroscopic scale one assumes that the current source is the synchronized synaptic activity, produced by several geometrically aligned cells firing together. Common simplified source models at the microscopic scale are point or spherical sources, seen as monopoles, while at the macroscopic scale, dipolar sources allow simpler yet generally accurate modelling. Note that single monopolar sources do not exist, as the various current sources in the brain must cancel each other in order to respect charge conservation (as for the dipolar case, which is an approximation of a two monopoles situation). Consequently, accurate microscopic modelling implies collections of monopoles, often representing compartments of detailed neuron models (see (Einevoll et al., 2013) for a review, as well as e.g. (Lindén et al., 2010; Leski et al., 2013; Ness et al., 2016)).

Forward problem consists in estimating the potentials in space (thus at the measurement sites) given a source model and a propagation model ${ }^{1}$. Starting from Maxwell equations, there is an abundant literature both for the microscopic and macroscopic cases. The main difficulty consists in correctly modelling the propagation environment, i.e., the impedances between the current sources and the measured potentials. Most of the research efforts are directed towards the evaluation of the homogeneous/inhomogeneous isotropic/non-isotropic nature of the brain and head tissues or to their geometrical approximations (see (e.g. Bangera et al.,

\footnotetext{
${ }^{1}$ All along this paper, by propagation we mean electrical field propagation in the brain tissues and not axonal propagation.
} 
2010; Hofmanis et al., 2013)). In all these studies, the implicit assumption is that there is no frequency dependence of the propagation model (impedance), at least in the frequency range of interest. In other words, the environment is assumed as purely resistive (although it might have different conductivities depending on the spatial position and on the orientation of the electric field). Indirect confirmations for these hypothesis are provided by the extremely large inverse problem literature: starting from the measurements and assuming a resistive propagation, quite good source localizations are obtained, both at the microscopic scale (current source density - CSD methods, (e.g. Pettersen et al., 2012)) and at macroscopic scale (dipolar fit, e.g. (Caune et al., 2014)).Direct confirmation of the dominantly resistive environment was provided by (Logothethis et al., 2007), who used an injected controlled current source in a particular geometrical setup in order to asses brain tissue impedance (which was found to have a rather insignificant frequency dependency, at about $1 \mathrm{~dB}$ per decade). Other studies arrived to more or less similar conclusions: in (Gabriel et al., 1996) for example, even if the permittivity displays a high negative slope with respect to the frequency on the whole tested frequency band $(10 \mathrm{~Hz}$ to $20 \mathrm{GHz})$, its influence on the absolute impedance value (and on the its phase) is negligible below $1000 \mathrm{~Hz}$, as pointed out also in (Bédard and Destexhe, 2009) (note however that the measurement uncertainties are rather important below $1000 \mathrm{~Hz}$, and even bigger below $100 \mathrm{~Hz}$ (Gabriel et al., 1996)). Moreover, further works of the same authors (Gabriel et al., 2009) only consider the resistive part of the impedance (i.e., the conductivity). Roughly the same variations were observed in two recent in vivo studies (Wagner et al., 2014; Dowrick et al., 2015), except for very low frequencies, below $100 \mathrm{~Hz}$.

An alternative model was developed at microscopic scale by (Bédard et al., 2006; Bédard and Destexhe, 2009; Bédard and Destexhe, 2014). In brief, this model aims to take into account the ionic diffusion in the brain tissue and proposes a Warburg type impedance of the propagation medium instead of the purely resistive one. Unlike in the resistive models, the brain tissue is assumed to have a complex impedance, scaling in modulus as the square root of the frequency (which 
yields a slope of $10 \mathrm{~dB}$ per decade, much higher than in previous studies). The main motivation and indirect proof for this hypothesis is that it provides an explanation for the experimentally observed $1 / f$ frequency scaling of the meso and macroscopic signal power spectral densites (local field potentials, LFP) (Bédard and Destexhe, 2009; Bédard et al., 2010; Destexhe and Bédard, 2013). Recently, a more direct confirmation was proposed by (Gomes et al., 2016), which uses an controlled intra-cellularly injected current source in order to determine a socalled natural impedance from which one can potentially separate the membrane impedance and the extracellular impedance.

The aim of this paper is to investigate the possible frequency dependency of the electrical field generated by a dipolar current source, at different distances from the source, at macroscopic scales (in the human brain). In this sense, our approach is quite similar to the one proposed by (Logothethis et al., 2007). The main difference is that we take the problem to the whole head scale. This leads to two important consequences for the modelling: the geometry is different, as the measurement electrodes can be far from the current source site (avoiding thus possible saturation problems) and, moreover, we have to deal with extremely different tissues, with a priori different impedances (e.g. white/gray matter vs. skull bone). As it will be shown, this allows us to tackle the possible frequency dependence of the brain tissue impedance using only the measured potentials at different locations, less dependent on the precise spectrum of the injected current source.

\section{MATERIALS AND METHODS}

\section{Experimental setup}

Our experimental setup is very similar to the one presented in (Bangera et al., 2010). In brief, a current source is artificially inserted into the brain, generating thus an electrical field (electrical stimulation). Current source and sink are neighbouring contacts, placed on one of the multi-contact intra-cerebral electrodes (see generic representation fig. 1). Measuring contacts are placed on several other intra-cerebral electrodes and on the scalp surface. 
Potentials can then be measured at different points in the brain and on the scalp with respect to a reference electrode placed itself on the scalp, sufficiently far from the stimulation site (reference at 'infinite' distance).

The intra-cerebral stimulations were delivered during standard presurgical evaluation of pharmacoresistant epileptic patients at the University Hospital (CHU) Nancy, France (recording and stimulation devices from Micromed, Italy, electrodes from Dixi Microelectronics, France). The patients gave their informed consent and the protocol was approved by the ethics committee of the hospital.

The procedure used in this paper was applied on several stimulation sessions (different stimulations sites, amplitudes and patients). We present here the details for one patient, but the results and the conclusions are very similar for all tested data.

The patient was implanted with 9 multi-contact electrodes (right hemisphere), having in all 117 contacts inside the brain, between 10 and 18 collinear contacts by electrode. Twenty surface EEG electrodes were used simultaneously, placed according to the standardized 10-20 system. The recording followed a standardized protocol: two neighbouring sensors on one of the depth electrodes (here the most profound ones, situated in the lingual gyrus) were used for stimulation, creating thus a current dipole. In order to avoid cells and tissue polarization, the stimulation was biphasic, i.e., the polarity of the stimulation current dipole changed as follows: a positive pulse lasting $0.5 \mathrm{~ms}$ was followed by a silent period of 0.05 $\mathrm{ms}$ and by a negative pulse with the same amplitude and duration as the positive one. Thirty to 120 pulses were delivered with a 1 second period per stimulation session, the sessions lasting thus between 30 seconds and 2 minutes. The used amplitudes varied from one session to another according to the clinical protocol, from 0.5 to $3 \mathrm{~mA}$.

The remaining 115 intra-cerebral and 20 scalp sensors were used for recording, at a sampling rate of $2048 \mathrm{~Hz}$. The reference electrode was placed on the scalp, at FPz position from the international 10-20 system. Because of its position with respect of the stimulation site, the reference electrode can be safely consid- 
ered at 'infinite' distance, thus null potential. The patient was asleep during the protocol and did not display any clinical or electro-physiological signs (epileptic spikes or seizures).

We did not consider in this analysis the signals recorded by sensors situated on the stimulation electrode, in order to avoid possibly saturated signals and propagation along the interface between the electrode and the tissue or within the multi-contact electrode. Consequently, the set of working signals contains 107 intra-cerebral potentials and 20 scalp potentials.

\section{Signal processing}

Unlike scalp EEG recordings, the SEEG signals have a much better signal to noise ratio and they are almost free of classical artefacts encountered in EEG, such as eye-blinks and muscle activity.

On the other hand, regardless of the potential due to the stimulation, all electrodes (reference electrode included) also record background physiological activity. If, by definition, we assume that this activity is independent from the stimulation, then it can be cancelled out or at least reduced by averaging epochs of 1 second, centred on each stimulation pulse. Note that, if the background physiological activity is low frequency and shows some coherence in time (e.g. regulars alpha waves), it will not be necessarily completely cancelled by averaging.

The time instants of the stimulation pulses were detected as the highest amplitude peaks recorded on a sensor close to the stimulation site and visually verified. In order to avoid possible (small) jitter due to the finite sampling frequency, we upsampled ( 8 times, from 2048 to $16384 \mathrm{~Hz}$ ) the signals before alignment (a similar approach was used for spike alignment before clustering in (Quian Quiroga et al., 2004)). This upsampling, which is basically equivalent to an interpolation between samples, is done using a low pass filter applied after inserting zeros between the samples of the original signal (see (Crochiere and Rabiner, 1983) for the theoretical aspects of the method, implemented in Matlab ${ }^{\circledR}$ ). It is important to mention here that upsampling cannot recover frequencies higher than the original Nyquist frequency (i.e., half of the original sampling rate), and the spectral 
content of the upsampled signal above this frequency is purely artefactual. Note finally that, if necessary, the unknown reference potential can also be cancelled by beamforming, especially when no epoch averaging is done (Madhu et al., 2012; Ranta and Madhu, 2012). In this study, reference cancelling by beamforming was also tested, but it did not improve simple averaging results.

The remaining 127 averaged signals of $1 \mathrm{sec}$ duration can now be considered as the potentials $V_{i}$ due to the stimulation, plus some remaining background activities.

It is important to observe that, depending on the amplitude of the stimulation current, it might trigger some evoked activities of the neurons close or connected to the stimulation site. In other words, besides the possibly remaining background, the recorded signals might still show activities which are not the image of the injected current source but some physiological evoked activity secondarily generated by the stimulation. We tested several stimulation sessions with amplitudes from 0.5 to $3 \mathrm{~mA}$, and we present here two datasets, at $1 \mathrm{~mA}$ and $3 \mathrm{~mA}$. In the first case, no evoked activity appeared on any of the recording contacts, while in the second case a clear low-frequency pattern was generated by the stimulation, and it reached its maximum amplitude about $20 \mathrm{~ms}$ after the stimulation on several contacts situated in the fusiform gyrus, at about $2 \mathrm{~cm}$ from the stimulation site (1.2 $\mathrm{mV}$ peak to peak). Nevertheless, in both situations (apparent evoked activity or not), it is safe to consider that the physiological activity remaining after averaging is situated mainly in the low frequencies, while the stimulation has a much broader and higher frequency band.

The amplitude spectra of depth and surface potentials (modulus of the Fourier transforms), as well as the average respective spectra are superimposed figure 2. One can distinguish the stimulation spectra (roughly above $100 \mathrm{~Hz}$ ), mainly visible on the depth sensors, but also on the surface ones for the high amplitude injected current. Background low frequency activity remains quite important. 


\section{Biophysical model}

Resistive medium. There is an abundant literature on modelling the electromagnetic propagation in the human head tissues (see e.g. (Baillet et al., 2001; Hallez et al., 2007; Bangera et al., 2010)). Most references, both in forward and inverse modelling (i.e., estimate the measured potentials given the source, respectively estimate the source given the measurements) focus on the geometry of the problem (numerical modelling of the anatomy) and use purely resistive models for the different propagation mediums, including gray and white matters, cerebro-spinal liquid, skull bones or skin and muscles. The assumption is that, in the frequency range of interest (roughly below $1000 \mathrm{~Hz}$, most of the times even below $100 \mathrm{~Hz}$ ), the tissues do not have significant frequency dependent characteristics.

Starting thus from the quasi-static Maxwell equations, the potentials generated by a current source (point or spherical) in a homogeneous isotropic medium can be computed as (Hallez et al., 2007; Logothethis et al., 2007):

$$
V_{i}=\frac{1}{4 \pi \sigma} I f\left(\mathbf{r}_{i}, \mathbf{r}_{o}\right)
$$

where $\sigma$ is the medium conductivity, $I$ is the stimulation current and $f\left(\mathbf{r}_{i}, \mathbf{r}_{o}\right)$ is a function depending on the (relative) positions of the stimulation site (source, $\mathbf{r}_{o}$ ) and of the measuring site (sensor, $\mathbf{r}_{i}$ ). Commonly, $f($.$) varies in 1 / r_{i}$, with $r_{i}$ being the source-sensor distance $\left\|\mathbf{r}_{i}-\mathbf{r}_{o}\right\|$.

The two close monopoles setup can be reduced to a dipole by simple limited developments, in which case the $f($.$) function appearing in (1) will include the$ distance between the two monopoles (assumed negligible compared to $r$, the distance between the source and the measurement point/electrode position) and it will vary in $1 / r^{2}$ rather than $1 / r$, as for monopoles. Note that the dipolar formalism is the most widely used for modelling both forward and inverse problems at macroscopic scales, where indeed the electrodes are far from the neural generators.

Non-resistive medium. Although the resistive models proved to be successful in forward/inverse modelling, there is one intriguing question they do not explain, 
namely the power spectral density characteristics of the LFP/EEG signals, which often display a $1 / f$ behaviour. In an attempt to tackle this issue, relatively recent works introduced a more complex modelling of the propagation medium, taking into account mainly the ionic diffusion and thus showing a frequency dependency. We will not detail here the models proposed in the different cited publications (Bédard et al., 2006; Bédard and Destexhe, 2009; Bédard et al., 2010; Bédard and Destexhe, 2014). The main result that interests us here is the general potential model for monopolar source in an isotropic medium, given by eq. (16) in (Bédard and Destexhe, 2009). Assuming that the position of the source is at the origin and due to the spherical symmetry of the problem,

$$
V_{i}(\omega)=\frac{I(\omega)}{4 \pi} \int_{r_{i}}^{\infty} \frac{1}{r^{\prime 2}} \frac{1}{\sigma\left(\omega, r^{\prime}\right)+i \omega \varepsilon\left(\omega, r^{\prime}\right)} d r^{\prime}
$$

where $r_{i}$ is the distance between the source and the measuring point ( $i^{\text {th }}$ sensor).

For an homogeneous medium, the conductivity and the permittivity do not depend on the position in space, thus on $r^{\prime}$, so they will factor out of the integral (2). Let $\gamma(\omega)=\sigma(\omega)+i \omega \varepsilon(\omega)$ the complex conductivity of the medium, including both resistive and reactive components. Then, after integrating (2), one obtains:

$$
V_{i}(\omega)=\frac{1}{4 \pi \gamma(\omega) r_{i}} I(\omega)
$$

or, for more general source configurations (e.g. dipoles, see above eq. (1)),

$$
V_{i}(\omega)=\frac{1}{4 \pi \gamma(\omega)} f\left(\mathbf{r}_{i}, \mathbf{r}_{o}\right) I(\omega)
$$

The complex impedance writes then

$$
Z_{i}(\omega)=\frac{1}{4 \pi \gamma(\omega)} f\left(\mathbf{r}_{i}, \mathbf{r}_{o}\right)
$$

and is similar to equation (17) from (Bédard and Destexhe, 2009) after integration.

The significant difference between models (1) and (4) is the complex and frequency dependent conductance $\gamma(\omega)$. As for the resistive case, it is a medium 
property ${ }^{2}$ and it is independent from the distance between the source and the measuring site (or orientation, for the dipolar case). Conversely, the position dependence given by $f\left(\mathbf{r}_{i}, \mathbf{r}_{o}\right)$ is independent from the medium physics.

We consider here, as in (Logothethis et al., 2007), that the current is injected between two electrodes (source/sink). In our setup, they are neighbouring sensors of a multi-sensors electrode. Consequently, seen from far measuring sensors, the source can be modelled as a current dipole, placed at position $\mathbf{r}_{o}$, having an orientation $\mathbf{c}_{o}$ along the stimulation electrode and an amplitude $I(\omega)$. Equation (4) writes then:

$$
V_{i}(\omega)=Z_{i}(\omega) I(\omega)
$$

where $Z_{i}(\omega)$ embed the geometry of the problem (i.e., the positions of the dipole and of the measuring electrode, $\mathbf{r}_{o}$ and $\mathbf{r}_{i}$ respectively, and the orientation of the dipole $\mathbf{c}_{o}$ ) and medium characteristics $\gamma(\omega)$, factorized separately (see eq. 5).

\section{Current source spectrum}

Stated differently, the measured potentials spectra are equal to the injected current spectrum weighted by $Z_{i}(\omega)$, that is by the (complex) conductance $\gamma(\omega)$ and some frequency independent gain. Evaluating this conductance can then naturally be done by imposing a specific spectrum to the current source (white noise or sinusoidal for example, as in (Logothethis et al., 2007; Gomes et al., 2016)). Specifically, the global impedance between the source and the $i$-th sensor writes:

$$
Z_{i}(\omega)=\frac{V_{i}(\omega)}{I(\omega)}
$$

If $I(\omega)$ and the geometry of the problem $f\left(\mathbf{r}_{i}, \mathbf{r}_{o}\right)$ are known, then $Z_{i}$ and next $\gamma(\omega)$ can be directly estimated from the measurement $V_{i}(\omega)$ using (7) and (5). Moreover, if the injected current is white noise (i.e., has a flat spectrum, $I(\omega)=c t$.),

\footnotetext{
${ }^{2}$ See (Bédard and Destexhe, 2009) for further developments of (5) depending on the medium characteristics.
} 
$Z_{i}(\omega)=k V_{i}(\omega)$, meaning that the spectrum of the measured potential is similar, up to a multiplicative constant, to the medium impedance spectrum.

Even if the current is not white, the same approach can be safely used if $I(\omega)$ is non-null for all frequencies, as long it is known. In our experimental setup, we are constrained by the clinical protocols and clinically approved devices. First, the injected current is not white. Still, as we know the injected pattern, we can easily compute its spectrum: it is broadband and it has significant values up to several $\mathrm{kHz}$ (unsurprisingly, considering that it is very brief in time). But on the other hand, the recording device has a very low sampling frequency, in this case fixed at $2048 \mathrm{~Hz}$, and thus an anti-aliasing filter included in the acquisition chain (2nd order low-pass filter at $500 \mathrm{~Hz}$, according to the manufacturer). While this anti-aliasing filter and sampling frequency are not a problem for physiological macroscopic EEG signals used in medical routine, the stimulation spectrum is too broad, and it remains too broad even after the anti-aliasing filter, easily above the Shannon frequency $(1024 \mathrm{~Hz})$. Consequently, sampling it at $2048 \mathrm{~Hz}$ highly and unpredictably distorts the signal, thus making it impossible to use in (7).

More precisely, the stimulation pattern writes:

$$
i(t)=\left\{\begin{array}{cl}
I_{\max }, & t \in\left(0, t_{1}\right) \\
0, & t \in\left(t_{1}, t_{1}+t_{p}\right) \\
-I_{\max }, & t \in\left(t_{1}+t_{p}, 2 t_{1}+t_{p}\right)
\end{array}\right.
$$

with $t_{1}$ the duration of the positive/negative pulse and $t_{p}$ the silent period between them (in our case 0.5 and $0.05 \mathrm{~ms}$ respectively). The Fourier transform will then be:

$$
I(\omega)=-\frac{1}{j \omega}\left(\mathrm{e}^{-j \omega t_{1}}\left(1+\mathrm{e}^{-j \omega t_{p}}\right)-1-\mathrm{e}^{-j \omega\left(2 t_{1}+t_{p}\right)}\right)
$$

The amplitude spectrum (up to $2048 \mathrm{~Hz}$, the sampling frequency of our instrumentation) is given figure 3. Superimposed on the same figure, we show the spectrum of the filtered version of the stimulation due to the anti-aliasing acquisition filter (2nd order low-pass at $500 \mathrm{~Hz}$ ). As it can be seen, significant energy remains located in high frequencies, above Nyquist frequency $(1024 \mathrm{~Hz})$.

We propose next an alternative approach. 


\section{Impedance ratio estimation}

We assume in this development that $I(\omega)$ is non-null for all frequencies, but not necessarily known. We assume also that we have several measuring electrodes, situated at different distances/angles from the injected current dipole position. In particular, electrodes $i$ and $j$ (positions $\mathbf{r}_{i}$ and $\mathbf{r}_{j}$, sufficiently far from $\mathbf{r}_{o}$ ) record the potentials $V_{i}(\omega)$ and $V_{j}(\omega)$, with respect to an external reference. Furthermore, we are not interested by gain differences (see the very complete study (Bangera et al., 2010) for attenuation evaluation of the potential depending on the distance and on the anisotropy of the medium), but only by the frequency dependence.

The ratio between $V_{i}(\omega)$ and $V_{j}(\omega)$ writes then:

$$
H_{i j}(\omega)=\frac{V_{i}(\omega)}{V_{j}(\omega)}=\frac{Z_{i}(\omega)}{Z_{j}(\omega)}
$$

In principle, if $\mathbf{r}_{i} \neq \mathbf{r}_{j}$, the impedances should also be different, and their ratio should vary depending on the their respective positions:

$$
\frac{Z_{i}(\omega)}{Z_{j}(\omega)}=f\left(\mathbf{r}_{o}, \mathbf{r}_{i}, \mathbf{r}_{j}, \omega\right)
$$

According to the factorization proposed by (4), the frequency should vanish if the sensors are placed inside the same homogeneous medium, as $\gamma(\omega)$ is the same, thus the ratio (9) should be flat in $\omega$.

Conversely, if the ratio (9) is the same for all $\omega$ (i.e., , the difference between impedances $Z_{i}$ and $Z_{j}$ is limited to the gain, constant for all frequencies), one can conjecture that the medium is the same for both electrodes $i$ and $j$.

One must note at this point that the previous argumentation holds only if $Z_{i}(\omega)$ and $Z_{j}(\omega)$ are dominated by the tissue impedance, i.e., if the electrode impedances are small compared to the tissue (in the opposite case, the ratio will be almost constant whatever the brain impedance, presuming that the different electrodes have the same impedances). Also, it is important to have a very high internal

impedance of the current generator and non-null current values for all explored 
frequencies, i.e., a broadband current source (in the opposite case, the ratio (8) will be undefined, $0 / 0$ ). Considering the stimulation pattern described above (very brief, thus large spectrum), this assumption can be safely accepted, at least for frequencies above $100 \mathrm{~Hz}$.

\section{RESULTS}

\section{Experimental findings}

Among the intra-cerebral signals, the potential recorded in the fusiform gyrus (at about $2 \mathrm{~cm}$ from the stimulation dipole, in the lingual gyrus) was chosen for normalization, as it has the best signal to noise ratio in the frequency band of interest (roughly above $100 \mathrm{~Hz}$, where the remaining background and the possible evoked activity are negligible and where the stimulation is sufficiently energetic).

Let this signal be $V_{j}(\omega)$. All $V_{i}(\omega)$ (both intra-cerebral and scalp recorded potentials) were normalized with respect to $V_{j}(\omega)$ to obtain $H_{i j}(\omega)(8)$. The results (modulus) are presented fig. 4.

A first observation is that, below $100 \mathrm{~Hz}$, the results are difficult to interpret. First, because the energy of the stimulation signal is low in this band, so the ratio $H_{i j}(\omega)$ is badly conditioned (see argumentation above). Next (and linked to the first argument), this is the range of the classical brain background activities, possibly not completely canceled out by epoch averaging. As these activities can be different from one recording site to another, the ratio can significantly vary. On the other hand, above $100 \mathrm{~Hz}$, one can assume that most of the signals' energy is due to the stimulation, at least for the depth intra-cerebral electrodes, so we will focus on this part of the spectrum.

For surface electrodes, muscular artefacts can be important up to at least 250 $\mathrm{Hz}$ (van Boxtel, 2001); for a high amplitude stimulation (fig. 4, right panel, dashed curve) the effect is less visible, but for the low amplitude stimulation, the surface electrodes are very likely to be perturbed by the EMG and thus difficult to interpret (fig. 4, left panel, dashed curve). Another possible cause is simply the noisier 
nature of surface recordings (the electrode-gel-skin impedance, electromagnetic noise, ....).

With this considerations in mind, we can still note that the impedance ratios (8) are flat above $\sim 200 \mathrm{~Hz}$ or even $100 \mathrm{~Hz}$ for the stronger injected current. This observation can be more precisely quantified by computing the slopes of the lines obtained by fitting first order polynomials on the impedance ratios (9), between 100 and $1000 \mathrm{~Hz}$. For example, for the SEEG impedance ratios during the 3 $\mathrm{mA}$ stimulation (light grey curves in figure 4, right panel), the mean slope is 2.8 $\mathrm{dB}$, with a standard deviation of $3 \mathrm{~dB}$. Among the 107 sensors, 93 have spectra yielding impedance ratios with slopes between +5 and $-5 \mathrm{~dB}$, which we define as "flat". When computing the mean and the standard deviation for these 93 slopes, the results are much less dispersed: the mean slope between 100 and $1000 \mathrm{~Hz}$ is 2 $\mathrm{dB}$ and the standard deviation is $1.5 \mathrm{~dB}$. In order to illustrate the arguments above, the slopes are graphically presented figure 5 for a $3 \mathrm{~mA}$ stimulation. The marked points indicate the signals (sensors) $V_{i}(\omega)$ for which the slopes are outside the \pm 5 $\mathrm{dB}$ interval, thus cannot be considered flat.

These signals (an interval of 10 seconds) are displayed in the bottom panel of figure 5, together with the $V_{j}$ signal used for normalization (first row). As it can be seen, there is almost no trace of stimulation on the 14 (=107-93) signals, meaning that the impedance ratio for these sensors is not reliable.

The previous observations hold for surface EEG signals (same stimulation amplitude) and for lower stimulation amplitudes also. For completeness, the results are summarized in table 1.

The results reported above lead to two conclusions: first, the factorization in (4) holds, that is the effects dues to the medium frequency characteristics and those dues to the source-sensors geometry can be decoupled. Second and most important, the medium characteristics seem similar for surface and depth electrodes. This conclusion holds after propagation at different distances and through different mediums (gray and white matters, cerebro-spinal liquid, skull bones, skin), indicating that they have similar complex conductivities $\gamma(\omega)$ up to a gain. 


\begin{tabular}{l|ccc} 
& Mean (STD) & $\mathrm{N}_{f}$ & Mean $_{f}\left(\mathrm{STD}_{f}\right)$ \\
\hline SEEG $(3 \mathrm{~mA})$ & $2.8(3.0)$ & $93 / 107$ & $2.1(1.5)$ \\
EEG $(3 \mathrm{~mA})$ & $2.3(3.4)$ & $19 / 20$ & $3.0(1.2)$ \\
SEEG $(1 \mathrm{~mA})$ & $-0.4(9.8)$ & $87 / 107$ & $0.2(1.4)$ \\
EEG $(1 \mathrm{~mA})$ & $-5.1(5.6)$ & $12 / 20$ & $-1.5(1.4)$ \\
\hline
\end{tabular}

Table 1: Quantified indication of the flatness of the impedance ratios for different stimulation amplitudes and for depth and surface signals. Mean (STD) are the mean and standard deviation values obtained for the whole set of signals, while $\operatorname{Mean}_{f}\left(\operatorname{STD}_{f}\right)$ are the values for the signals yielding a "flat" impedance ratio (between $\pm 5 \mathrm{~dB}$ ). The number of flat impedance ratios is given by $N_{f}$, with respect to the total number of signals. Values for mean and standard deviation are in $\mathrm{dB}$.

Considering the different nature of the head tissues (bone vs. soft tissues mainly), this is highly unlikely, and the most plausible conclusion is that, in the frequency range 100 to $1000 \mathrm{~Hz}$, the macroscopic scale conductance is actually purely or overwhelmingly resistive, both inside the brain and in the other head tissues, confirming thus the conclusion of (Logothethis et al., 2007).

\section{Computational models}

A resistive propagation medium, as indicated by the previous results, implies that the LFP spectra is similar (up to the gain) to the current source spectra, in particular to synaptic currents. We propose below a family of possible fractional computational models of the synaptic currents, aiming to fit the observed $1 / f$ LFP spectrum. These models are fitted to "golden standard" kinetic models (Destexhe et al., 1998) and tested against established synaptic models from the literature ${ }^{3}$ and against simulated LFP data.

Assume a fractional system (Caponetto et al., 2010) of order $1 / 2$, which is basically similar to the Warburg impedances proposed in (Bédard and Destexhe,

\footnotetext{
${ }^{3}$ See Appendix for a brief presentation of these models
} 
2009; Gomes et al., 2016):

$$
H_{w}(s)=\frac{1}{1+\left(s \tau_{w}\right)^{1 / 2}}
$$

Its impulse response $h(t)$ can be obtained by inverse Laplace transform:

$$
h_{w}(t)=\frac{1}{\sqrt{\pi \tau_{n}}} t^{-1 / 2}-\frac{1}{\tau_{n}} \mathrm{e}^{t / \tau_{n}} \operatorname{erfc}\left(\sqrt{\frac{t}{\tau_{n}}}\right)
$$

where $\operatorname{erfc}(x)$ is the complementary error function:

$$
\operatorname{erfc}(x)=\frac{2}{\sqrt{\pi}} \int_{x}^{\infty} \mathrm{e}^{-t^{2}}
$$

A possible synapse model can then be the fractional system of order $3 / 2$ :

$$
H_{3 / 2}(s)=\frac{1}{1+s \tau} H_{w}(s)
$$

having an impulse response obtained by convolving the simple decaying exponential and (10):

$$
h_{3 / 2}(t)=h_{w}(t) * \frac{1}{\tau} \mathrm{e}^{-t / \tau}
$$

Although not analytically tractable (because of $h_{w}(t)$ ), one can obtain the result numerically for any causal system $t>0$. This $h_{3 / 2}(t)$ represents now the time course of the synaptic current (triggered by a Dirac pulse arriving in the presynaptic neuron) and basically replaces the $r(t)$ in (13) or the responses $h(t)$ from table 3 ). The two parameters ( $\tau$ and $\tau_{w}$ ) can be identified by fitting $h_{3 / 2}$ to the kinetic models from (Destexhe et al., 1998). Note that for GABA-B synapse, a third parameter is needed, corresponding to the time constant $\tau_{2}$ of the supplementary low-pass filter. The results of these fits are presented in figure 6 .

In order to have a quantitative evaluation, the relative errors between the computational models and the kinetic ones (norm of the error normalized by the norm of the kinetic response) are given table 2. Globally, as it can be seen and expected, less parameters are used, worse the fitting results. The $\alpha$-function fitting is consistently less accurate than the other three models, fractional included. This 
global impression need to be nuanced when trying to separate between fractional and multiple exponential fittings. Indeed, for rapid synapses (AMPA and GABAA), the exponential models perform better and, as a matter of fact, almost similar. For the slower (but simple kinetic) NMDA synapse, the fractional is better than the double exponential (both having thus the same number of parameters / time constants) but worse than the 3-exponential model (4 parameters). Finally, for the more complicated kinetics of GABA-B, the 2 parameter fractional model fits better than the others, including the 4 parameter model $h_{3}$.

\begin{tabular}{lcccc} 
Model $/$ Synapse & AMPA & GABA-A & NMDA & GABA-b \\
\hline fractional & 0.2071 & 0.1005 & 0.0936 & 0.1608 \\
\hline$\alpha$-function $h_{\alpha}$ & 0.2431 & 0.2970 & 0.3513 & 0.2588 \\
\hline $\begin{array}{l}\text { 2 exponentials } h_{2} \\
\text { (Dayan and Abbott, 2001) }\end{array}$ & 0.1184 & 0.0201 & 0.1414 & 0.2567 \\
\hline $\begin{array}{l}\text { (exponentials } h_{3} \\
\text { (Gerstner et al., 2014) }\end{array}$ & 0.0863 & 0.0201 & 0.0089 & 0.2299 \\
\hline
\end{tabular}

Table 2: Relative errors between the computational approximations and the respective kinetic models.

The influence of the fractional synaptic impulse response (12) on the LFP spectrum is illustrated by the simulation presented in figure 7 . This figure, which is to be compared with figure 4 in (Bédard and Destexhe, 2009) illustrates the spectral characteristics of the LFP, assumed to be the result of the propagation through a resistive medium of post-synaptic current sources. Clearly, a simple exponential model does not exhibit odd slopes of the PSD, while fractional models or multiple exponential models fit more or less the $1 / f$ real PSD characteristics reported in the literature. Note that, unlike in (Bédard and Destexhe, 2009), the propagation medium is here purely resistive. 


\section{DISCUSSION}

The experimental setup described here, using simultaneous depth and scalp recordings of a stimulation signal allowed to tackle the brain impedance evaluation problem in an original way. The aim was to confirm or infirm the different results one can find in the literature, namely resistive vs. complex (frequency dependent) impedance. If we consider only the intra-cerebral electrodes, we cannot decide if there exists a frequency dependence of the intra-cerebral medium described by some complex conductance $\gamma(\omega)$, because it factors out from the measured spectra ratio (8). Based only on these intra-cerebral measurements, the medium conductivity could be frequency dependent or purely resistive.

On the other hand, the spectra are also similar when considering the surface measurements. This implies that the same frequency weighting of the injected current spectrum is done for the depth and surface electrodes. In other words, one might neglect the differences between the tissues and assume that the whole propagation medium has the same complex conductivity $\gamma(\omega)$, except for the gain.

The results and conclusions reported here do not agree with microscopic scale findings, neither with the PSD of the synaptic related LFP, as for example reported in (Bédard and Destexhe, 2009), nor with the findings from (Gomes et al., 2016) (sub-threshold LFP measured after intracellular current injection). The explanations advanced in (Bédard and Destexhe, 2009) are based on the nature of the source: high intensity artificial sources (like in our case as well as in (Logothethis et al., 2007)) block the ionic diffusion by diminishing the ionic gradient and only the ohmic part of the impedance remains apparent. Indeed, in Logothethis in vivo experiments (Logothethis et al., 2007), the measuring electrodes were placed between the injecting electrodes, on the maximum current density line. In our setup though, the measuring electrodes were far from the current source, thus not affected by the local gradient due to the current injection. Moreover, even if locally the injected current was much higher that physiological sources, the potential generated on the far electrodes used in our experiments was well in the range of the physiological activities, i.e., having similar or smaller amplitudes than distantly 
generated physiologic epileptic peaks for example. In other words, even if diffusion is blocked near high intensity sources, it should not be blocked far from them, in the distant brain tissue.

In our opinion, other explanations are needed to conciliate between macroscopic impedance measurements and the microscopic findings reported in (Bédard and Destexhe, 2009; Gomes et al., 2016). A first possible explanation would be that the diffusive impedance proposed in the cited papers appears only in the immediate neighbourhood of the current sources, i.e., ionic channels on the membrane (synaptic or not). Consequently, at the microscopic scale, it dominates the overall impedance between the source and the measuring electrode. On the contrary, at a macroscopic scale, it is the resistive part that becomes predominant and the Warburg-like impedance can be neglected.

Other explanations are possible, involving not the medium but the intrinsic nature of the current generators, which might have a more complicated dynamics, either fractal (thus fractional), see (Liebovitch, 1989; Liebovitch and Krekora, 2002) or simply involving much richer channel openings and closings, modelled using sums of several exponentials (Colquhoun and Hawkes, 2009, chap. 18 in Sakmann \& Neher, Single-Channel Recordings). The latter explanation is to be linked to realistic neuron morphologies, with detailed compartmental modelling involving thus multiple current generators with different morphologies and spatial positions with respect to the recording site. Indeed, as shown by (Lindén et al., 2010) and further modelled by (Pettersen et al., 2014), more realistic neuron models yield different LFP spectra and spike morphologies through an "intrinsic dendritic filtering", depending on the position of the recording electrode and of course on the particular morphology of the neuron.

Note that, from a phenomenological point of view, the two types of source dynamics (i.e., fractal dynamics and sums of exponentials) are approximately equivalent, as any irrational transfer function (i.e., including fractional power of $\omega$ ) can be approximated (in any arbitrarily chosen frequency band) by a product of rational transfer functions with a dense interlacing of poles and zeros (Charef et al., 
1992), which corresponds to a sum of exponentials in the time domain.

We might finally conjecture that all the previous explanations, although proposing physically different mechanisms, are equivalent from a macroscopic phenomenological point of view. Indeed, ionic diffusion creates an accumulation of charges around open channels (Bédard and Destexhe, 2011), and this phenomenon might need to be considered for evaluating the potential generated by the opening/closing of the channel itself. Seen from a far away electrode, the current source includes multiple channels, having different positions and dynamics, together with their neighborhood. The resulting overall fractional dynamics can then be approximated by rather simple fractional computational models, with a reduced number of parameters.

\section{CONCLUSION}

This paper presents experimental results obtained in the in vivo human brain at a macroscopic scale, aiming to evaluate the impedance of the propagation medium. Using sensors placed in mediums having different impedances (depth and surface electrodes) we try to alleviate the apparent contradictory experimental results from the literature. Indeed, while classical models assume a purely resistive brain tissue (Logothethis et al., 2007), recent theories and results tend to suggest that the propagation medium in the brain, or at least in the cortex, has a frequency dependent impedance which can be factorized in a distance dependent part and a frequency dependent part varying as $1 / \sqrt{\omega}$, probably due to ionic diffusion (Bédard and Destexhe, 2009; Gomes et al., 2016).

Our experimental results show that, at macroscopic scale, the brain tissue (as well as the skull and the scalp) do not present frequency dependent conductivities in a frequency range between 100 and $1000 \mathrm{~Hz}$. This confirms classical resistive models thoroughly used in forward an inverse modelling at the macroscopic scale $^{4}$.

\footnotetext{
${ }^{4}$ It is nevertheless important to note that the the fact that the source localization part of the
} 
One might conjecture then that non-resistive effects appear at a microscopic scale, either near the current source (i.e., ionic channels on the neuron membrane or in the synapse) or even inside it, that is incorporated in its dynamics. Computationally, they might be modelled either as a sum of several exponentials, thus having several tuning parameters, or using fractional models.

\section{ACKNOWLEDGEMENTS}

The authors would like to thank Alain Destexhe for fruitful discussions and comments on the early versions of the manuscript, as well as Laurent Koessler, researcher at CRAN, and Dr. Sophie Colnat-Coulbois, neurosurgeon at the University Hospital in Nancy, for helping with the data.

\section{APPENDIX}

We start by briefly recalling the kinetic models proposed in (Destexhe et al., 1994, 1998), which we considered as golden standards for the simulations (see also (Colquhoun and Hawkes, 2009) for the theoretical justification of this formalism). Next, we also recall some classical synaptic computational models.

\section{Kinetic models}

Biophysically founded kinetic synapse models, very close to actual measurements, were proposed in (e.g. Destexhe et al., 1994, 1998). These models take into account detailed biochemical mechanisms for different synapse types (AMPA, NMDA, GABA-A, GABA-B). ${ }^{5}$

inverse problems (not to be mistaken for the time course/frequency content estimation) yields valid results is not an argument by itself in the favour of a resistive medium. Indeed, the localization results are not affected by the frequency dependent tissue impedance, as long as the factorization in (4) holds.

${ }^{5}$ For the detailed models, the reader is referred to the cited publications (Destexhe et al., 1994, 1998). 
The first three synapse types can be well approximated by simplified kinetics as proposed (Destexhe et al., 1998), which can be further described by one nonlinear differential equation (the non-linearity being related to the neuro-transmitter release, itself related to the action potential of the pre-synaptic neuron for the detailed models):

$$
I(t)=\bar{g} r(t)(V-E)
$$

where $I$ is the synaptic current, $\bar{g}$ is its maximal conductance, $V$ is the membrane potential of the postsynaptic neuron and $E$ is the respective reversal potential. The time dynamics is given by $r(t)$, modeled as:

$$
\frac{d r}{d t}=\alpha[T](1-r)-\beta r
$$

In (14), $[T]$ is a non-linear pulse of transmitter release and $\alpha$ and $\beta$ are synapse specific parameters.

The last synapse (GABA-B) has a more complicated kinetics, but it can also be modelled using 2 coupled differential equations, the first one similar to (13), the second one being a simple first order linear differential equation:

$$
\frac{d s}{d t}=K_{2} r-K_{4} s
$$

Note that this last equation corresponds to first order low-pass filter with a time constant $\tau_{2}=1 / K_{4}(s(t)$ being the filtered version of $r(t))$.

\section{Computational models}

In the literature one can find a lot of phenomenological/computational synaptic models, from simple decaying exponentials as the one used in (Bédard and Destexhe, 2009), passing through alpha functions (Rall, 1967), to multiple exponentials (Dayan and Abbott, 2001; Gerstner et al., 2014) ${ }^{6}$. A summary of the time

\footnotetext{
${ }^{6} \mathrm{We}$ do not intend to present an exhaustive list, but rather to illustrate the diversity of the models
} 
expressions (impulse responses $h(t))$ and corresponding transfer functions $(H(s)$, the Laplace transforms of $h(t))$ is given table 3.

As said previously, under the hypothesis that the medium is resistive, the time courses and consequently the spectral characteristics of the synaptic currents determine the LFP power spectrum. As it can readily be seen, they computational models from table 3 are all exponentials and their spectral characteristics do not match the measured LFP as reported in (Bédard and Destexhe, 2009). 


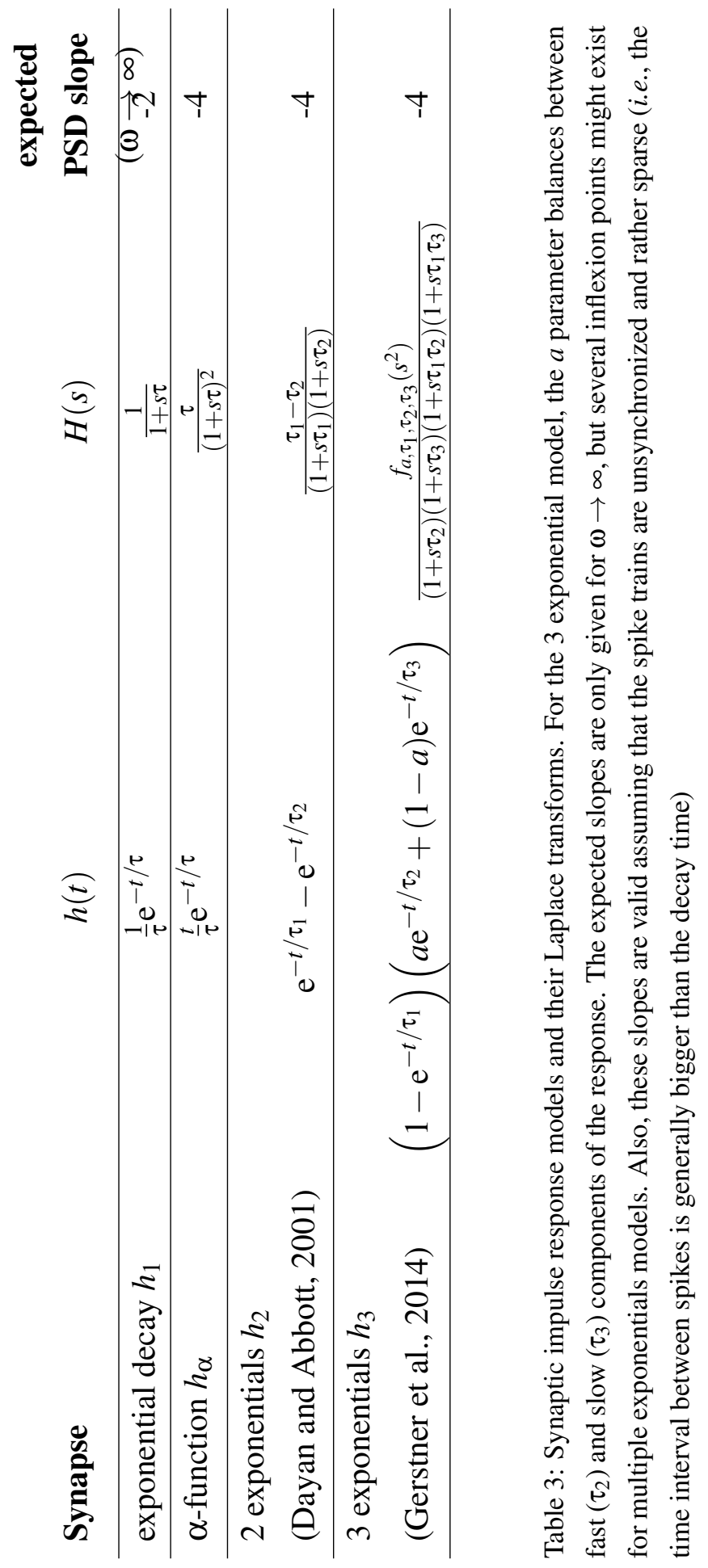




\section{REFERENCES}

Baillet S, Mosher J, Leahy R (2001). Electromagnetic brain mapping. IEEE Signal Proc Mag 18(6):14-30.

Bangera NB, Schomer DL, Dehghani N, Ulbert I, Cash S, Papavasiliou S, Eisenberg SR, Dale AM, Halgren E (2010) Experimental validation of the influence of white matter anisotropy on the intracranial EEG forward solution. J Comput Neurosci 29(3):371-387.

Bédard C, Destexhe A (2009) Macroscopic models of local field potentials and the apparent 1/f noise in brain activity. Biophys J 96:2589-2603.

Bédard C, Destexhe A (2011) A generalized theory for current-source density analysis in brain tissue. Phys Rev E 84:041909.

Bédard C, Destexhe A (2014) Mean-field formulation of Maxwell equations to model electrically inhomogeneous and isotropic media. J Electromagn Anal Appl 6:296-302.

Bédard C, Kröger H, Destexhe A (2006) Model of low-pass filtering of local field potentials in brain tissue. Phys Rev E 73(5):051911.

Bédard C, Rodrigues S, Roy N, Contreras D, Destexhe A (2010) Evidence for frequency-dependent extracellular impedance from the transfer function between extracellular and intracellular potentials. J Comput Neurosci 29(3):389403.

Caponetto R, Dongola G, Fortuna L, Petras I (2010) Fractional Order Systems: Modeling and Control Applications. World Scientific Publishing Company.

Caune V, Ranta R, Le Cam S, Hofmanis J, Maillard L, Koessler L, Louis-Dorr V (2014) Evaluating dipolar source localization feasibility from intracerebral SEEG recordings. NeuroImage 98:118-133. 
Charef A, Sun HH, Tsao YY, Onaral B (1992) Fractal system as represented by singularity function. IEEE T Automat Contr 37(9):1465-1470.

Colquhoun D, Hawkes AG (2009) The principles of the stochastic interpretation of ion-channel mechanisms. In: Sackmann B, Neher E (Eds.), Single-Channel Recording. Springer, 397-482, 2nd edition.

Crochiere RE, Rabiner LR (1983) Multirate Digital Signal Processing. PrenticeHall, Englewood Cliffs, NJ.

Dayan P, Abbott L (2001) Theoretical Neuroscience: Computational and Mathematical Modeling of Neural Systems (Computational Neuroscience). MIT Press, Cambridge, Massachusetts.

Destexhe A, Bédard C (2013) Local field potential. Scholarpedia. http: //www . scholarpedia.org/article/Local_field_potential

Destexhe A, Mainen ZF, Sejnowski TJ (1994) Synthesis of models for excitable membranes, synaptic transmission and neuromodulation using a common kinetic formalism. J Comput Neurosci 1:195-230.

Destexhe A, Mainen ZF, Sejnowski TJ (1998) Kinetic models of synaptic transmission. In: Koch, C., Segev, I. (Eds.), Methods in Neuronal Modeling, 2nd Edition. MIT Press, Cambridge, MA, 1-25.

Dowrick T, Blochet C, Holder D (2015) In vivo bioimpedance measurement of healthy and ischaemic rat brain: implications for stroke imaging using electrical impedance tomography. Physiol Meas 36(6):1273-1282.

Einevoll GT, Kayser C, Logothetis NK, Panzeri S (2013) Modelling and analysis of local field potentials for studying the function of cortical circuits. Nat Rev Neurosci 14(11):770-785.

Gabriel C, Peyman A, Grant EH (2009) Electrical conductivity of tissue at frequencies below $1 \mathrm{MHz}$. Phys Med Biol 54(16):4863-4878. 
Gabriel S, Gabriel C, Lau R (1996) The dielectric properties of biological tissues: II. Measurements in the frequency range $10 \mathrm{~Hz}$ to $20 \mathrm{GHz}$. Phys Med Biol 41(11):2251-2269.

Gerstner W, Kistler WM, Naud R, Paninski L (2014) Neuronal Dynamics. Cambridge University Press, online book. http://neuronaldynamics .epfl.ch/ online/index.html

Gomes JM, Bédard C, Valtcheva S, Nelson M, Khokhlova V, Pouget P, Venance L, Bal T, Destexhe A (2016) Intracellular Impedance Measurements Reveal Non-ohmic Properties of the Extracellular Medium around Neurons. Biophys J 110(1):234-46.

Hallez H, Vanrumste B, Grech R, Muscat J, De Clercq W, Vergult A, D'Asseler Y, Camilleri K, Fabri S, Van Huffel S, Lemahieu I (2007) Review on solving the forward problem in EEG source analysis. J Neuroeng Rehabil 4:46.

Hofmanis J, Caspary O, Louis-Dorr V, Ranta R, Maillard L (2013) Denoising depth EEG signals during DBS using filtering and subspace decomposition. IEEE T Bio-Med Eng 60(10):2686-2695.

Leski S, Lindén H, Tetzlaff T, Pettersen KH, Einevoll GT (2013) Frequency Dependence of Signal Power and Spatial Reach of the Local Field Potential. PLoS Comput Biol 9(7).

Liebovitch LS (1989) Testing fractal and markov models of ion channel kinetics. Biophys J 55(2):373-377.

Liebovitch LS, Krekora P (2002) The physical basis of ion channel kinetics: the importance of dynamics. In: Layton HE, Weinstein AM (Eds.), Membrane Transport and Renal Physiology. IMA V Math 129:27-52.

Lindén H, Pettersen KH, Einevoll GT (2010) Intrinsic dendritic filtering gives low-pass power spectra of local field potentials. J Comput Neurosci 29(3):423444. 
Logothethis N, Kayser C, Oeltermann A (2007) In vivo measurement of cortical impedance spectrum in monkeys: Implications for signal propagation. Neuron 55:809-823.

Madhu N, Ranta R, Maillard L, Koessler L (2012) A unified treatment of the reference estimation problem in depth EEG recordings. Med Biol Eng Comput 50(10):1003-1015.

Ness TV, Remme MWH, Einevoll GT (2016) Active subthreshold dendritic conductances shape the local field potential. J Physiol 594 (13):3809-3825.

Pettersen KH, Lindén H, Dale AM, Einevoll GT (2012) Extracellular spikes and current-source density. In: Brette R, Destexhe A (Eds.), Handbook of Neural Activity Measurement. Cambridge University Press.

Pettersen KH, Lindén H, Tetzlaff T, Einevoll GT (2014) Power laws from linear neuronal cable theory: Power spectral densities of the soma potential, soma membrane current and single-neuron contribution to the EEG. PLOS Comput Biol 10(11):1-26.

Quian Quiroga R, Nadasdy Z, Ben-Shaul Y (2004) Unsupervised spike detection and sorting with wavelets and superparamagnetic clustering. Neural comput 16(8):1661-1687.

Rall W (1967) Distinguishing theoretical synaptic potentials computed for different soma-dendritic distributions of synaptic input. J Neurophysiol 30(5):11381168 .

Ranta R, Madhu N (2012) Reference Estimation in EEG: Analysis of Equivalent Approaches. IEEE Signal Proc Let 19:12-15.

van Boxtel A (2001) Optimal signal bandwidth for the recording of surface EMG activity of facial, jaw, oral, and neck muscles. Psychophysiology 38:22-34. 
Wagner T, Eden U, Rushmore J, Russo CJ, Dipietro L, Fregni F, Simon S, Rotman S, Pitskel NB, Ramos-Estebanez C, Pascual-Leone A, Grodzinsky AJ, Zahn M, Valero-Cabré A (2014) Impact of brain tissue filtering on neurostimulation fields: A modeling study. NeuroImage 85:1048-1057. 


\section{Figures}

Figure 1: Example of electrode implantation (stimulation contacts in black) and schematic representation of an intracerebral electrode (dimensions in $\mathrm{mm}$ )

Figure 2: Superimposed spectra of the recorded signals for different electrodes (depth: left panels / surface: right panels) and different stimulation amplitudes (1 mA: top panels / $3 \mathrm{~mA}$ : bottom panels). Averaged spectra are superimposed in black. Note the different amplitude scales for depth and surface spectra

Figure 3: Theoretical stimulation spectrum $I(\omega)$ before (solid line) and after anti-aliasing filter (dotted line). The vertical line represents the Nyquist frequency, half of the sampling frequency

Figure 4: Impedance ratios considering the stimulation source at $1 \mathrm{~mA}$ (left panel) and $3 \mathrm{~mA}$ (right panel). Clear grey curves correspond to intracerebral sensors, while dark grey curves correspond to surface EEG sensors. Averaged ratios are superimposed in black, dashed line for the surface signals

Figure 5: Up: the slopes of the fitted lines for the 107 SEEG computed impedance ratios ( $3 \mathrm{~mA}$ stimulation). The marked values are outside the $\pm 5 \mathrm{~dB}$ interval (14 values out of 107). Bottom: the normalization signal $V_{j}$ and the 14 signals $V_{i}$ yielding impedance ratios $\frac{V_{i}(\omega)}{V_{j}(\omega)}$ with slopes $>5$ $\mathrm{dB} / \mathrm{dec}$ (absolute values)

Figure 6: Normalized synaptic impulse responses: kinetic models (Destexhe et al., 1998) in black dashed line vs. computational approximations (fractional, $\alpha$-functions, double (Dayan and Abbott, 2001) and triple exponentials (Gerstner et al., 2014)). AMPA synapse (top left), NMDA (top right), GABA-A (bottom left) and GABA-B (bottom right)

Figure 7: Synaptic activities LFP. A: raster plot of 8 simulated units (Poisson processes with variating $\lambda(t)$; B-E: Power spectral densities (PSD, normalized) of the generated LFP, using the $\delta$ functions given by the raster convolved with different synaptic responses: simple exponential decay ( $\tau=10 \mathrm{~ms}$, as in (Bédard and Destexhe, 2009)) (B); fractional NMDA model (C); double exponential (Dayan and Abbott, 2001) (C) and triple exponential (Gerstner et al., 2014) (E). The lines on figure (B) have slopes of 0 and -2 , while on figures (C) to (E) they have slopes of -1 and -3 , more consistent with the real observed PSD of the LFP 


\section{Tables}

Table 1: Quantified indication of the flatness of the impedance ratios for different stimulation amplitudes and for depth and surface signals. Mean (STD) are the mean and standard deviation values obtained for the whole set of signals, while $\operatorname{Mean}_{f}\left(\mathrm{STD}_{f}\right)$ are the values for the signals yielding a "flat" impedance ratio (between $\pm 5 \mathrm{~dB}$ ). The number of flat impedance ratios is given by $N_{f}$, with respect to the total number of signals. Values for mean and standard deviation are in $\mathrm{dB}$

Table 2: Relative errors between the computational approximations and the respective kinetic models

Table 3: Synaptic impulse response models and their Laplace transforms. For the 3 exponential model, the $a$ parameter balances between fast $\left(\tau_{2}\right)$ and slow $\left(\tau_{3}\right)$ components of the response. The expected slopes are only given for $\omega \rightarrow \infty$, but several inflexion points might exist for multiple exponentials models. Also, these slopes are valid assuming that the spike trains are unsynchronized and rather sparse (i.e., the time interval between spikes is generally bigger than the decay time) 

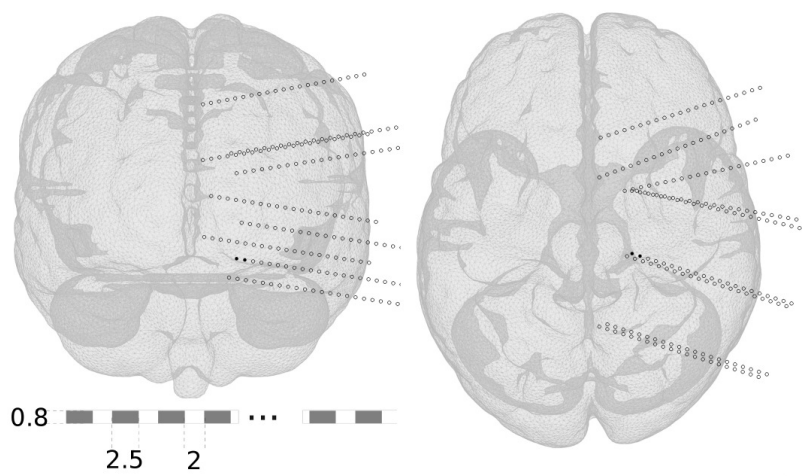

Figure 1:

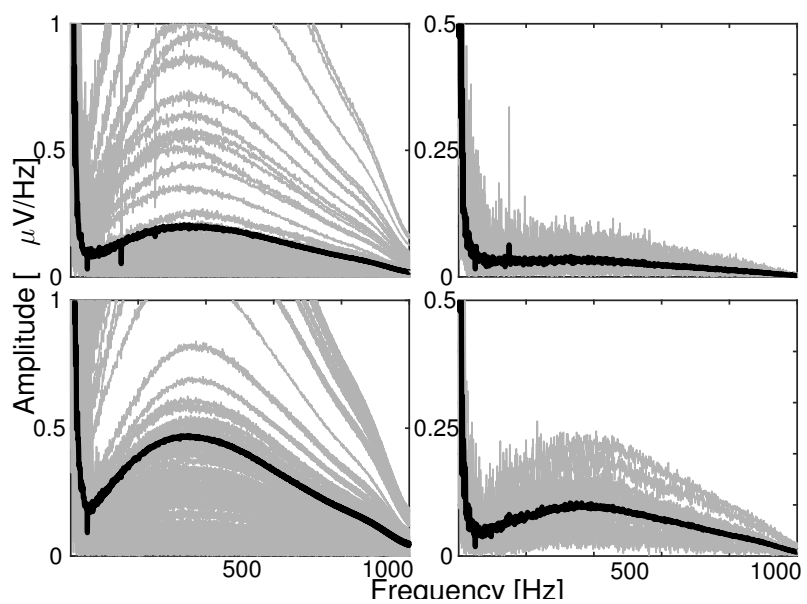

Figure 2: 


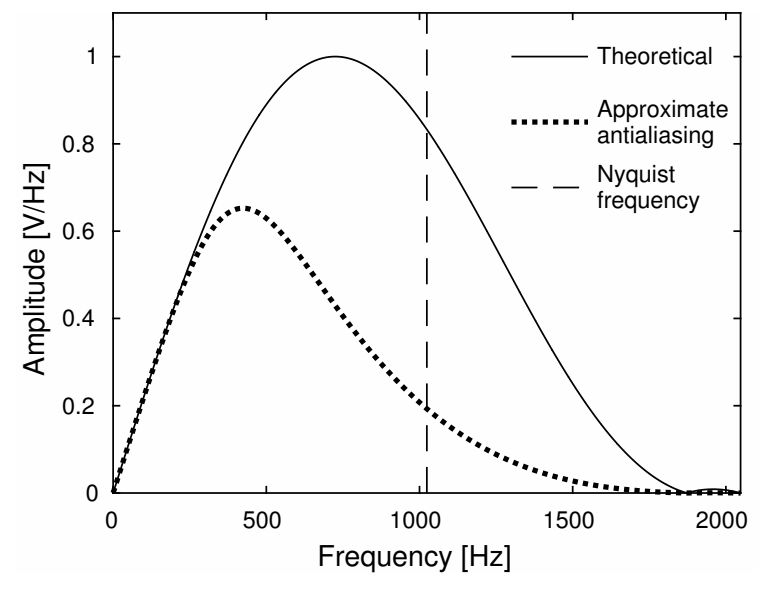

Figure 3:

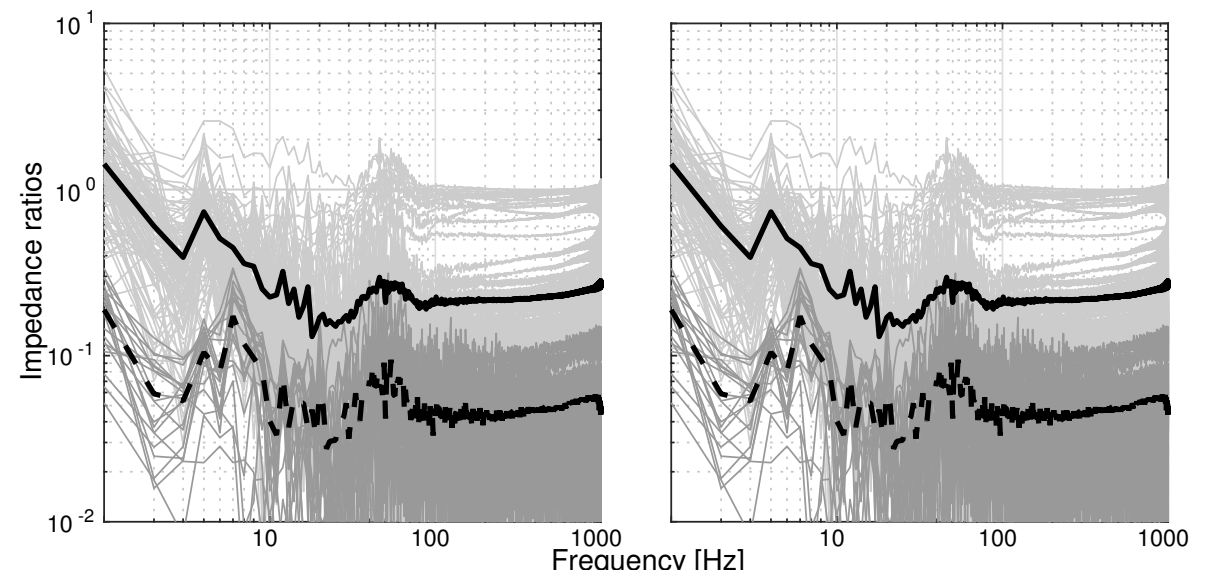

Figure 4: 

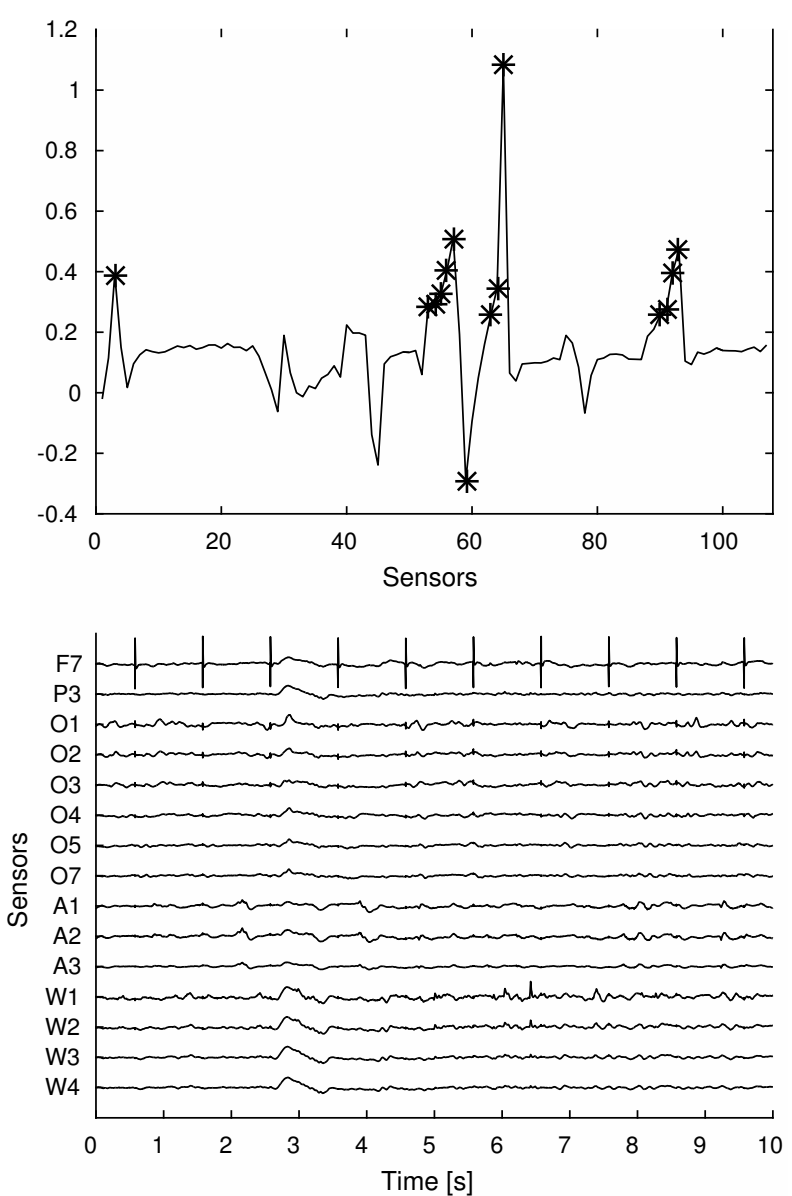

Figure 5: 

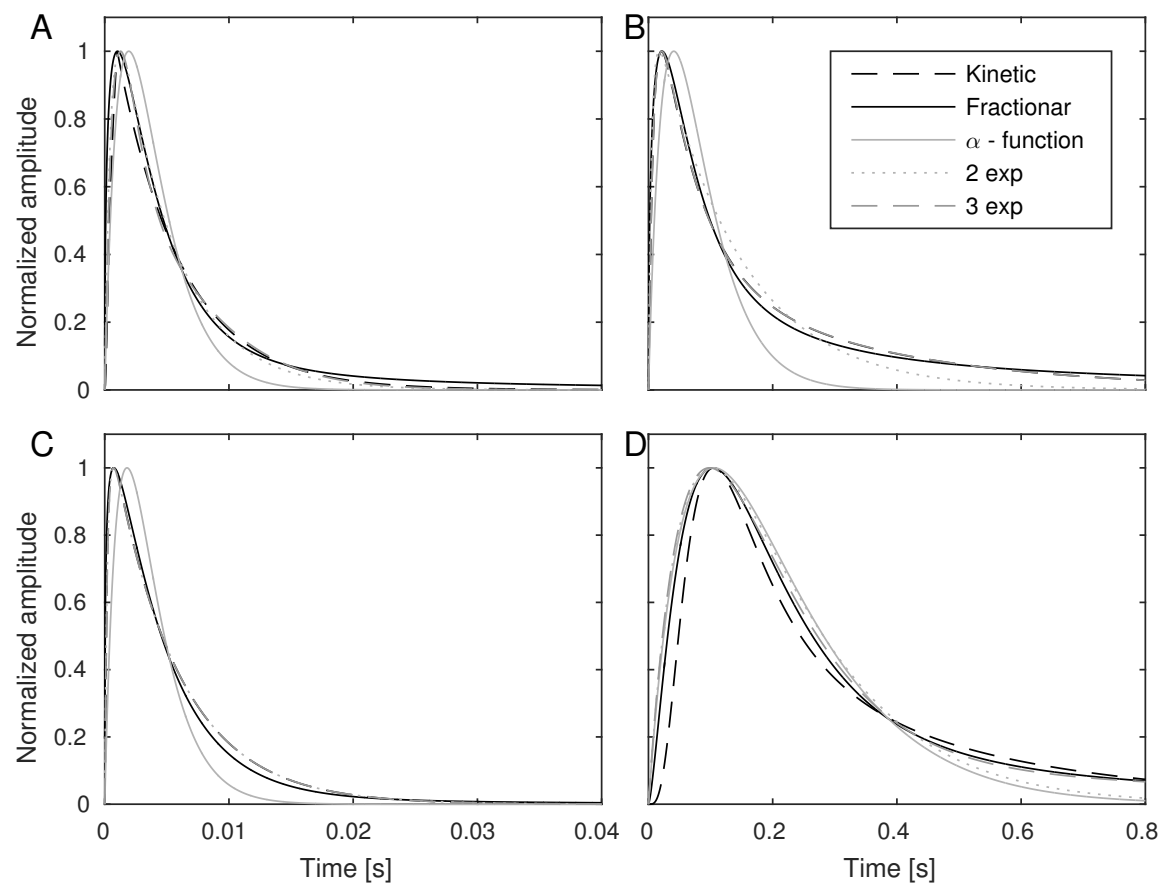

Figure 6: 

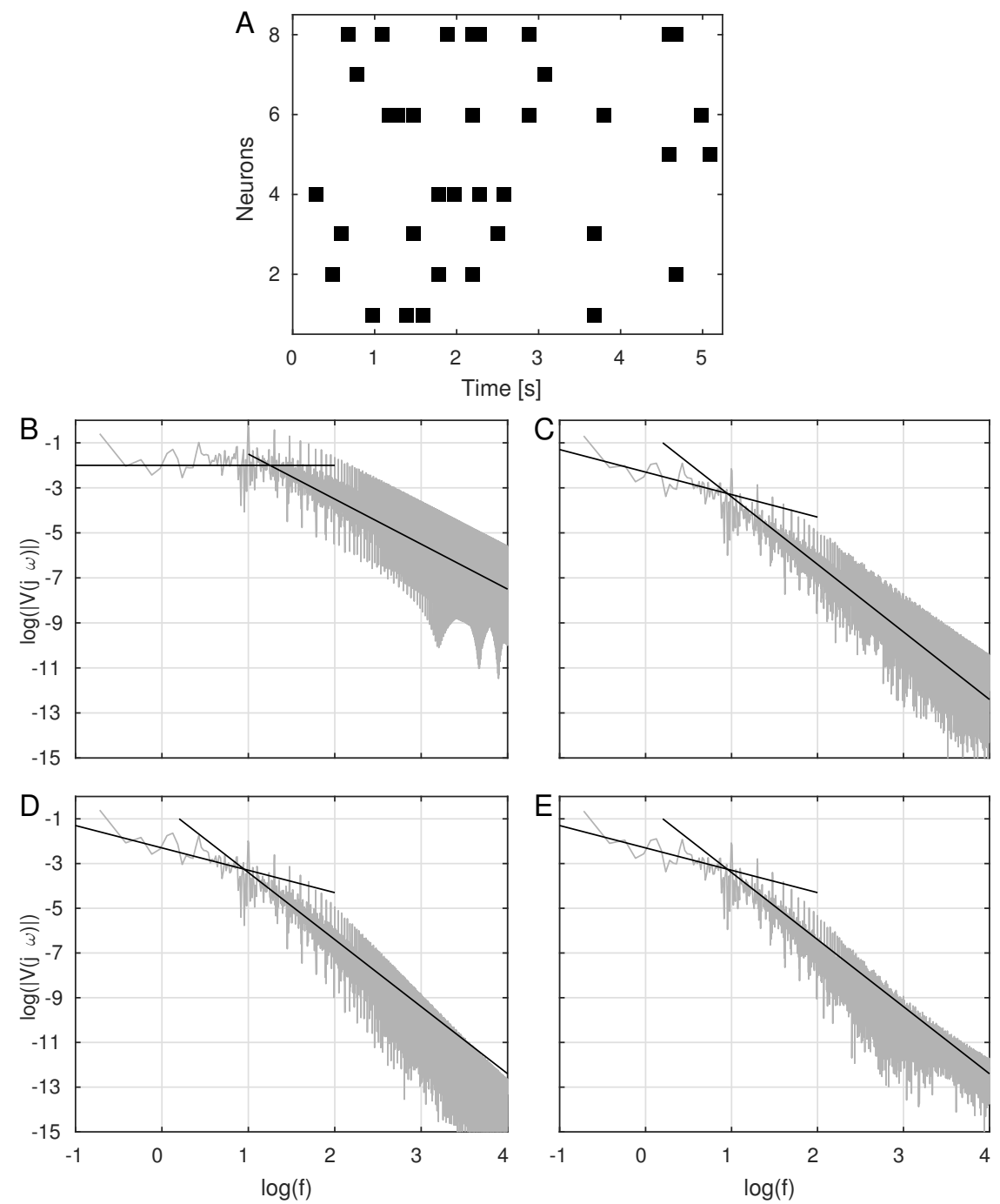

Figure 7: 